\title{
Is surgical repair of an asymptomatic groin hernia appropriate? A review
}

\author{
B. van den Heuvel · B. J. Dwars · D. R. Klassen • \\ H. J. Bonjer
}

Received: 30 August 2010 / Accepted: 16 January 2011 / Published online: 5 February 2011

(C) Springer-Verlag 2011

\begin{abstract}
Purpose Groin herniorrhaphy is the most common operation performed by general surgeons. Annually, more than 20 million groin hernias are repaired worldwide. The general approach towards groin hernias is surgical repair regardless of the presence of symptoms. The rationale to recommend surgery for asymptomatic groin hernias is prevention of visceral strangulation. The goal of this review is to evaluate the appropriateness of surgery in patients with asymptomatic groin hernias.

Methods The review was based on an extensive literature search of Pubmed, Medline and the Cochrane Library.

Results The risk of incarceration is approximately 4 per 1,000 patients with a groin hernia per year. Risk factors for incarceration are age above 60 years, femoral hernia site and duration of signs less than 3 months. Morbidity and mortality rates of emergency groin hernia repair are higher in patients who are older than 49 years, have a delay between onset of symptoms and surgery of more than $12 \mathrm{~h}$, have a femoral hernia, have nonviable bowel and have an ASA-class of 3 or 4 . The recurrence rate after tension-free mesh repair in the management of emergency groin hernias is comparable to that of elective repair. There is no difference in pain and quality of life after elective repair compared to watchful waiting. There is no advantage in cost-effectiveness of elective repair compared to watchful waiting.
\end{abstract}

B. van den Heuvel $(\bowtie) \cdot$ B. J. Dwars

Slotervaartziekenhuis, Louwesweg 6,

1066 EC Amsterdam, The Netherlands

e-mail: baukjevdh@yahoo.com

D. R. Klassen · H. J. Bonjer

QE II Health Sciences Center,

Dalhousie University, Halifax, BC, Canada
Conclusion Watchful waiting for asymptomatic groin hernias is a safe and cost-effective modality in patients who are under 50 years old, have an ASA class of 1 or 2, an inguinal hernia, and a duration of signs of more than 3 months.

Keywords Inguinal · Hernia $\cdot$ Asymptomatic $\cdot$ Therapy

\section{Introduction}

Groin herniorrhaphy is the most common operation performed by general surgeons. Annually, over 20 million groin hernias are repaired worldwide [1]. In the United States, 800,000 groin hernia repairs were performed in 2003 [2]. Groin hernia repairs account for $10-15 \%$ of all general surgical procedures [2].

The general policy towards groin hernias is surgical repair regardless of the presence of symptoms. The rationale to recommend surgery for asymptomatic groin hernias is to prevent visceral incarceration and subsequently ischemia (strangulation). There is a low threshold to propose surgery in patients with groin hernias because the repair is considered safe and effective, and is associated with a low morbidity. However, little is known about the natural history of untreated groin hernias, and morbidity of groin hernia repair appears underestimated [3]. The goal of this study was to review outcomes of surgical and conservative approaches in patients with a groin hernia, and to determine the appropriateness of surgery in patients with an asymptomatic or minimal groin hernia.

\section{Methods}

This systematic review was based on an extensive literature search of Pubmed, Medline and the Cochrane Library. The 
following query was used to identify relevant articles: (hernia AND inguin*) AND (incarcerat* OR strangulat* OR incident* OR risk OR asymp* OR (watchful* AND wait*)).

Relevant articles were first selected by title and then confirmed by reading the corresponding abstracts. Missing articles were identified by hand searches of the reference list of the leading articles and from articles brought to the attention by experts. All articles were analyzed by one reviewer.

Definitions of incarceration and strangulation vary widely. The following definitions are used throughout this review: an incarcerated hernia is an irreducible hernia and a strangulated hernia is an irreducible hernia with signs of visceral ischemia or gangrene. The term "emergency hernia" refers to hernias that require emergency repair, including both incarcerated and strangulated hernias.

\section{Incidence}

The incidence of groin hernias in adults increases with age [4]. It is estimated that the incidence of groin hernias is 0.7 per 1,000 per year between the age of 45-64 years rising to 1.5 per 1,000 per year over the age of 75 [5]. Some reports suggest that the incidence of groin hernias increases sixfold during lifetime in males [6]. In a large epidemiologic study in the Oxford region, 1.3 per 1,000 people per year were admitted for herniorrhaphy [7]. In Sweden, a similar incidence of 1.8 per 1,000 people per year was found [8].

The two most common types of groin hernias are inguinal and femoral hernias. Femoral hernias account for less than $10 \%$ of all groin hernias, while $30-50 \%$ of these hernias present as emergencies due to incarceration or strangulation [5, 8-13]. Inguinal hernias are 9-12 times more common in men than in women, whereas femoral hernias are 4 times more common in women [4, 7-9]. Strangulated inguinal hernias are more common in males and strangulated femoral hernias are more common in females [14].

The presenting symptom of a groin hernia in two-thirds of all patients is either discomfort or pain in the groin [15]. One-third of all patients is asymptomatic at presentation and presents with the sign of a non-tender bulge in the groin. When a groin hernia cannot be diagnosed by physical examination, imaging is indicated. Ultrasonography reveals groin hernias at sensitivity and specificity rates greater than 90\% [16]. MRI is most commonly employed in differentiating the causes of groin pain in the absence of a hernia [1].

Morbidity and mortality of elective groin hernia repair

The most common short-term complications after groin hernia surgery are pain, hematoma, seroma and wound infection. The most common long-term complication is chronic pain in the groin and recurrence of the hernia. The short-term morbidity rates reported in the literature are shown in Table 1 . The average morbidity rate of groin hernias is $8 \%$.

Chronic pain is generally defined as pain or discomfort lasting longer than 3 months after surgery [17]. The incidence of chronic pain after hernia repair reported in the

Table 1 Type, morbidity and mortality in elective groin hernia repair

\begin{tabular}{|c|c|c|c|c|c|}
\hline Study & $N$ & Inguinal hernia & Femoral hernia & Morbidity & Mortality \\
\hline Palumbo et al. 1954 [26] & 604 & & & $36(5.9 \%)$ & $1(0.1 \%)$ \\
\hline Williams et al. 1966 [40] & 222 & $222(100 \%)$ & $0(0.0 \%)$ & $59(27 \%)$ & $4(2 \%)$ \\
\hline Ponka et al. 1974 [12] & 200 & $140(70 \%)$ & $60(30 \%)$ & $75(38 \%)$ & $1(0.5 \%)$ \\
\hline Tingwald et al. 1982 [39] & 44 & $43(98 \%)$ & $1(2 \%)$ & $8(18 \%)$ & $0(0.0 \%)$ \\
\hline Nehme 1983 [52] & 1,044 & & & $273(26 \%)$ & $14(1 \%)$ \\
\hline Allen et al. 1987 [53] & 51 & $49(96 \%)$ & $2(4 \%)$ & & $0(0.0 \%)$ \\
\hline Lichtenstein 1987 [25] & 6,321 & $6,125(94 \%)$ & $196(3 \%)$ & $331(5 \%)$ & $2(0.04 \%)$ \\
\hline Lewis et al. 1989 [34] & 97 & & & $21(21.6 \%)$ & $0(0.0 \%)$ \\
\hline Oishi et al. 1991 [13] & 1,777 & $1,758(99 \%)$ & $19(1 \%)$ & $116(6.5 \%)$ & $0(0.0 \%)$ \\
\hline Primatesta et al. 1996 [7] & 27,937 & & & & $366(1.3 \%)$ \\
\hline Nilsson et al. 1997 [8] & 4,879 & $4,137(85 \%)$ & $173(3.5 \%)$ & $286(6 \%)$ & $3(0.07 \%)$ \\
\hline Haapaniemi et al. 1999 [54] & 17,061 & $16,086(94 \%)$ & $375(6 \%)$ & & $24(0.1 \%)$ \\
\hline Bay-Nielsen et al. 2001 [21] & 25,148 & & & & $55(0.2 \%)$ \\
\hline Ohana et al. 2004 [38] & 200 & & & $21(10.5 \%)$ & $0(0.0 \%)$ \\
\hline Total & 85,585 & $28,560(93 \%)^{\mathrm{a}}$ & $826(2.7 \%)^{b}$ & $1,226(8.0 \%)^{\mathrm{c}}$ & $470(0.5 \%)$ \\
\hline
\end{tabular}

${ }^{a}$ Total percentage of numbers published $(28,560 / 30,555)$

b Total percentage of numbers published $(826 / 30,555)$

c Total morbidity rate of numbers published $(1,226 / 15,388)$ 
literature varies widely. This wide range is due to different techniques being used and to a large variability in the description of pain [18]. Chronic pain after suture repair was reported by Cunningham et al. [19], with $63 \%$ of the patients having pain in the groin area after 1 year, decreasing to $54 \%$ after 2 years. The incidence of chronic pain after open tension-free mesh and laparoscopic repair is lower but still considerable. These rates in literature after open tension-free mesh repair vary from 13 to $37 \%$ [20-23]. The incidence of chronic pain after laparoscopic repair is similar and varies from 10 to $30 \%$ [20-23]. A recent multicenter randomized controlled trial by Eklund et al. [17] compared the incidence of chronic pain after both open tension-free mesh (Lichtenstein) and laparoscopic (TEP) repair. A total of 1,370 patients were included and randomized. After 5 years, $9 \%$ of the patients reported any kind of pain after laparoscopic repair versus $19 \%$ after Lichtenstein repair. The percentage of patients that reported pain interfering with daily activities was $2 \%$ after TEP repair versus $3.5 \%$ after Lichtenstein repair. A similar study by Langeveld et al. [24] also compared TEP and Lichtenstein repair. After 1 year, $25 \%$ of the patients reported postoperative pain after TEP repair compared to $29 \%$ after Lichtenstein repair. These rates are higher than those reported by Eklund and colleagues, but it is not clear whether these patients were impaired in their daily activities. These results show the difficulty in interpreting postoperative pain rates and comparing them. A universal approach to assessing chronic pain after herniorrhaphy was proposed by Kehlet et al. [17] and should be implemented to interpret incidences of chronic pain and to compare results.

Recurrence rates after herniorrhaphy depend mostly on the surgical technique used for repair. Recurrence rates after suture repair have been reported as high as $62 \%[4,25$, 26]. When Lichtenstein introduced the open tension-free mesh repair in the 1970s, recurrence rates were greatly reduced to $0-10 \%[25,27-30]$. In the early 1990 s, laparoscopic techniques using a tension-free mesh were developed for groin hernia repair. The recurrence rates after laparoscopic repair are comparable to those after open tension-free mesh repair, and range between 2 and $4 \%[20,22$, $24,31]$.

The mortality rate after elective groin hernia repair is low. The causes of death in elective groin hernia repair are mostly cardiovascular, advanced cancer, sepsis and pulmonary diseases. Table 1 provides an overview of mortality rates found in the literature; these average $0.5 \%$. Nilsson et al. [32] studied mortality rates after elective groin hernia repair in a time period of 13 years in Sweden. These rates were defined in a standardized mortality ratio (SMR), comparing observed deaths of operated patients to expected deaths considering the age and gender of the population in Sweden. They found that the mortality rate after elective groin hernia repair does not transcend the expected mortality, implying that elective groin hernia repair is a safe and low-risk operation.

Risk of incarceration and strangulation

Accurate determination of the actual risk of incarceration and strangulation of a groin hernia is complex because of a paucity of studies on the actual incidence of these events. An indirect parameter of incarceration and strangulation is the rate of emergency groin hernia repair, assuming that emergency repairs are done in patients with either incarcerated or strangulated groin hernias. Table 2 summarizes emergency ratios reported in the literature. Almost $7 \%$ of all hernia repairs are emergency repairs.

In Columbia, an epidemiologic study was done to assess the incidence of incarceration and strangulation [6]. A random stratified sample was taken of the civilian population to assess the prevalence of groin hernias. Regionally, all emergency repairs were observed during a 5-year period. The number of emergency repairs was divided by the prevalence of groin hernias to provide an estimation of the incidence of incarceration and strangulation. The overall risk of incarceration and strangulation was 3.6 per 1,000 male adults and 5.4 per 1,000 female adults with a groin hernia per year.

Several risk factors have been identified that may predict incarceration and strangulation. Acknowledgement of these

Table 2 Emergency groin hernia repairs

\begin{tabular}{lrc}
\hline Study & $N^{\mathrm{a}}$ & Emergency repair \\
\hline Williams et al. 1966 [40] & 270 & $48(18 \%)$ \\
Kauffman et al. 1970 [42] & 966 & $102(10.5 \%)$ \\
Palumbo et al. 1971 [4] & 3,572 & $86(2.4 \%)$ \\
Ponka et al. 1974 [12] & 200 & $34(17 \%)$ \\
Tingwald et al. 1982 [39] & 62 & $18(29 \%)$ \\
Nehme 1983 [42] & 1,279 & $235(18 \%)$ \\
Lewis et al. 1989 [34] & 116 & $19(16 \%)$ \\
Gallegos et al. 1991 [11] & 476 & $34(7 \%)$ \\
Oishi et al. 1991 [13] & 1,859 & $82(4.4 \%)$ \\
Primatesta et al. 1996 [7] & 30,675 & $2,738(9 \%)$ \\
Nilsson et al. 1997 [8] & 4,879 & $284(6 \%)$ \\
Nilsson et al. 1998 [55] & 12,542 & $719(6 \%)$ \\
Haapaniemie et al. 1999 [54] & 18,170 & $1,109(6.1 \%)$ \\
Hair et al. 2000 [56] & 5,506 & $294(5 \%)$ \\
Bay-Nielsen et al. 2001 [21] & 26,304 & $1,156(4 \%)$ \\
Kulah et al. 2001 [14] & 3,010 & $385(13 \%)$ \\
Malek et al. 2004 [3] & 532 & $38(7.1 \%)$ \\
Ohana et al. 2004 [38] & 2,331 & $67(2.9 \%)$ \\
Total & $1,03,537$ & $7,448(6.6 \%)$ \\
\hline
\end{tabular}

a Total number of groin hernia repairs 
Table 3 Mean age, hernia type, morbidity and mortality in emergency groin hernia repair

\begin{tabular}{|c|c|c|c|c|c|c|c|}
\hline Study & $N$ & Mean age & Inguinal hernia & Femoral hernia & Morbidity & Mortality & Bowel resection \\
\hline Williams et al. 1966 [40] & 48 & $>60$ & $48(100 \%)$ & $0(0.0 \%)$ & $25(52 \%)$ & $6(13 \%)$ & \\
\hline Kauffman et al. 1970 [42] & 102 & & $102(100 \%)$ & $0(0.0 \%)$ & & $10(10 \%)$ & \\
\hline Andrews 1981 [9] & 190 & 68 & 82 & 72 & & $19(11 \%)$ & \\
\hline Tingwald et al. 1982 [39] & 18 & 76.9 & $15(83 \%)$ & $3(17 \%)$ & $10(56 \%)$ & $4(22 \%)$ & $2(11 \%)$ \\
\hline Nehme 1983 [52] & 235 & $>65$ & & & $131(56 \%)$ & $18(8 \%)$ & $26(11 \%)$ \\
\hline Allen et al. 1987 [53] & 64 & 76 & $46(72 \%)$ & $18(28 \%)$ & & $4(7 \%)$ & \\
\hline McEntee et al. 1987 [36] & 48 & & & & & $4(9 \%)$ & $13(27 \%)$ \\
\hline Lewis et al. 1989 [34] & 19 & 78.6 & & & $11(58 \%)$ & $2(10 \%)$ & \\
\hline Brasso et al. 1989 [35] & 114 & & $87(76 \%)$ & $27(24 \%)$ & & $3(3 \%)$ & $13(11 \%)$ \\
\hline Oishi et al. 1991 [13] & 82 & 50.3 & $67(82 \%)$ & $15(18 \%)$ & $17(21 \%)$ & $5(6.1 \%)$ & $25(30 \%)$ \\
\hline Gallegos et al. 1991 [11] & 34 & 65.5 & $22(65 \%)$ & $12(35 \%)$ & & & $6(18 \%)$ \\
\hline Askew et al. 1992 [57] & 54 & & $13(24 \%)$ & $29(54 \%)$ & & $2(4 \%)$ & $15(28 \%)$ \\
\hline Primatesta et al. 1996 [7] & 2,738 & 58 & & & & $175(6.4 \%)$ & \\
\hline Nilsson et al. 1997 [8] & 284 & & & & & $10(3.5 \%)$ & $19(6.7 \%)$ \\
\hline Rai et al. 1998 [10] & 181 & & & & & $11(6 \%)$ & \\
\hline Haapaniemi et al. 1999 [54] & 1,109 & & $905(82 \%)$ & $204(18 \%)$ & & $34(3.1 \%)$ & \\
\hline Kulah et al. 2001 [37] & 189 & 72.1 & $108(57 \%)$ & $42(22 \%)$ & $48(25 \%)$ & $10(5 \%)$ & $36(19 \%)$ \\
\hline Bay-Nielsen et al. 2001 [21] & 1,156 & 73 & & & & $81(7 \%)$ & \\
\hline Kulah et al. 2001 [14] & 385 & 55.1 & $222(58 \%)$ & $69(18 \%)$ & $75(19.5 \%)$ & $11(2.9 \%)$ & $53(14 \%)$ \\
\hline Kurt et al. 2003 [41] & 102 & 53 & $62(60.8 \%)$ & $13(12.8 \%)$ & $17(17 \%)$ & $2(1 \%)$ & $16(15.7 \%)$ \\
\hline Alvarez et al. 2004 [33] & 147 & 70 & $70(48 \%)$ & $77(52 \%)$ & $61(41.5 \%)$ & $5(3.4 \%)$ & $19(12.9 \%)$ \\
\hline Malek 2004 [3] & 38 & 76 & $19(50 \%)$ & $19(50 \%)$ & $13(34 \%)$ & $4(11 \%)$ & $13(34 \%)$ \\
\hline Ohana et al. 2004 [38] & 67 & 69.1 & & & $16(23.9 \%)$ & $4(6.0 \%)$ & $3(4.5 \%)$ \\
\hline Total & 7,404 & & $1,868(68 \%)^{\mathrm{a}}$ & $600(22 \%)^{\mathrm{b}}$ & $424(32 \%)^{\mathrm{c}}$ & $424(5.8 \%)^{\mathrm{d}}$ & $259(14 \%)^{\mathrm{e}}$ \\
\hline
\end{tabular}

a Total percentage of numbers published $(1,868 / 2,743)$

b Total percentage of numbers published $(600 / 2,743)$

c Total morbidity rate of numbers published $(424 / 1,330)$

d Total mortality rate of numbers published $(424 / 7,370)$

e Total rate of bowel resections based on numbers published $(259 / 1,797)$

risk factors would help in allocating a patient to a high- or low-risk group, and to decide for which patient elective repair is desirable and which patient can be treated conservatively. Many different risk factors for incarceration and strangulation have been analyzed in the literature.

Femoral hernias incarcerate and strangulate significantly more frequently than inguinal hernias [8, 12-14, 33]. Table 3 summarizes details and outcomes of emergency groin hernia repair, including hernia site. An eight-fold increase in the ratio of femoral hernias in emergency repair (22\% in emergency repair vs. $2.7 \%$ in elective repairs) is observed. With older age, the risk of incarceration and strangulation increases for both genders [7, 14, 34]. Brasso et al. [35] found a peak incidence in the age group of 61-80 years, and McEntee et al. [36] found a peak incidence in the age group of 70-80 years. The cumulative probability of incarceration and strangulation increases over time; 3 months after the onset of signs of an inguinal hernia, the cumulative probability of incarceration and strangulation is $2.8 \%$. This percentage rises to $4.5 \%$ after 2 years. For femoral hernias, the cumulative probability is much higher: $22 \%$ at 3 months and $45 \%$ at 21 months [11]. The cumulative probability increases most in the first period after the onset of signs of a groin hernia. Two-thirds of patients that present with an emergency hernia have had their groin hernia for less or equal to 1 year [10]. In the elective group, two-thirds of patients had their hernia for longer than 1 year.

In conclusion, risk factors for incarceration or strangulation are age, hernia site and duration of signs.

Morbidity and mortality of emergency groin hernia repair

Emergency groin hernia repair is associated with higher morbidity and mortality rates than elective groin hernia repair $[8,11,13,34,37,38]$. An overview of these rates 
reported in the literature is summarized in Table 3. The average morbidity rate is $32 \%$ and the average mortality rate is $5.8 \%$, compared to 8 and $0.5 \%$, respectively, for elective repair.

In Sweden, Nilsson et al. [32] studied the mortality rates after emergency groin hernia repair using the standardized mortality ratio (SMR). The observed deaths after emergency groin hernia repair transcends the expected deaths significantly when the age of the patient is above 49 years, when the patient has an ASA class above 2, in the case of a femoral hernia, and when bowel resection was required during surgery. These result are confirmed by other studies [14, 35, 37, 39, 40].

Table 3 shows that $14 \%$ of all emergency hernias contain necrotic bowel, necessitating bowel resection. Bowel resections are more common in patients above the age of 65 years, late hospitalization, coexisting diseases and femoral hernias [3, 9, 12-14, 33, 35, 37, 41, 42]. The morbidity and mortality rate after emergency hernia repair necessitating bowel resection is significantly higher compared to no bowel resection [14]. Bekoe [43] related the presence of nonviable bowels in strangulated hernias to duration of symptoms. The incidence of nonviable bowels requiring bowel resection increases 15 -fold within $24 \mathrm{~h}$, rising from $3 \%$ when duration of symptoms was less than $12 \mathrm{~h}$ to $50 \%$ when duration of symptoms was over $24 \mathrm{~h}$. Andrews [7] showed that late hospitalization in general correlates with higher morbidity and mortality rates after emergency groin hernia repair. A mortality rate of $1 \%$ was found when patients were hospitalized within the first $24 \mathrm{~h}$ after onset of symptoms, increasing to $21 \%$ after a delay of more than $47 \mathrm{~h}$.

In conclusion, age over 49 years, late hospitalization, femoral hernia site, nonviable bowel and ASA-class above 2 increase the morbidity and mortality rate after emergency repair significantly.

\section{Recurrence rate after emergency groin hernia repair}

Different techniques are used in cases of emergency hernia [35]. The use of a prosthetic mesh in emergency repair is presumed to lead to mesh-related complications such as an increased risk of infection [44, 45]. Recent studies have shown that the use of a tension-free mesh in emergency groin hernia repair is not associated with higher morbidity rates. Elsebae et al. [44] compared tension-free mesh repair to the Bassini repair in emergency groin hernias in a randomized controlled trial. Patients with preoperative peritonitis, an inflammatory hernia and ischemic necrosis necessitating bowel resection were excluded from the study. Postoperative complication rates did not differ between the two groups. During follow-up of 22 months, no recurrence was observed in the tension-free mesh group compared to three recurrences in the Bassini group $(P<0.001)$. Bessa et al. [45] compared the use of mesh in elective and emergency groin hernia repair. Twenty-five patients with a mean age of 60 years, and ASA class 1 or 2, presented with an emergency groin hernia. All emergency hernias were repaired with a tension-free mesh according to Lichtenstein. The results were compared to a matching group of 25 patients who underwent elective tension-free mesh hernia repair. Results show that the tension-free mesh repair in the management of emergency groin hernias is not associated with a higher rate of complications, including recurrence rate, compared to its use in the elective setting.

\section{Watchful waiting}

In 2006, two randomized controlled trials about watchful waiting versus elective repair in asymptomatic inguinal hernias were published. Fitzgibbons et al. [46] randomized 720 patients to either a tension-free mesh repair group or a watchful waiting group. The main outcomes were pain and discomfort interfering with usual activities measured in the physical component score of the Short Form-36, Version 2. After 2 years, both groups reported less pain than at baseline. The amount of change from baseline in pain while at rest, during normal activities, and during work or exercise did not differ between the groups. The reduction in perception of pain unpleasantness was significantly greater for patients in the surgical repair group. The mean follow-up was 3.2 years. During follow-up, two cases $(0.6 \%)$ of incarceration were observed in the watchful waiting group, resulting in an accident rate of 1.8 per 1,000 patients per year. Twenty-three percent of the patients assigned to the watchful waiting group did receive surgical repair within 2 years, mostly because of increasing pain and discomfort. Seventeen percent of the patients assigned to the surgery group did not undergo surgical repair in 2 years, with no reason given in the majority of cases.

Stroupe et al. [47] assessed costs, quality-adjusted lifeyears (QALY) and cost-effectiveness at 2-years follow-up of the same group of subjects as the above-mentioned trial of Fitzgibbons et al. [46]. QALY incorporates healthrelated quality of life and medical outcomes into a single measure [47]. The average direct costs for the surgical repair group were significant higher than the watchful waiting group with a difference of US $\$ 1,831$ ( $\$ 7,875$ vs. $\$ 6,044)$. The patients in the surgical repair group had a 0.031 higher mean QALY than the patients in the watchful waiting group. The incremental cost-effectiveness ratio, which refers to the cost per additional QALY was US \$59,065/QALY. A cost per QALY of approximately US \$50,000 is generally viewed as a reasonable cutoff for public funding of a medical procedure in the United States [48]. 
O'Dwyer et al. [49] randomized 160 patients with an asymptomatic or minimally symptomatic inguinal hernia to elective tension-free mesh repair or to observation. The primary outcome was pain at rest and movement measured by the use of the $100 \mathrm{~mm}$ visual analogue pain score (VAS), and general health status measured by the Short Form-36 (SF-36) at 12 months. At 12 months, no significant differences were found between both groups with regards to pain at rest or at movement. General health status increased for both the surgical and the observational arm over 12 months. The general health status in the surgical arm increased significantly more compared to the observational arm. Despite improvement in general health in the surgical arm, no significant difference in QALY was found at 12 months. Another outcome measured was cost to the Health Service. Results show a difference in direct cost of $£ 402$ per patient for the operation group compared to the observation group. One case of incarcerated hernia (1.3\%) was observed in the wait-and-see group during follow-up of 12 months. At 12 months, $19 \%$ of the patients in the observational arm crossed over to surgery, mostly due to increase of pain.

Summarizing, both studies compared surgical repair to watchful waiting in the treatment of inguinal hernias. Fitzgibbons showed that both groups reported less pain after 2 years than at baseline. The reduction in perception of pain unpleasantness was significantly greater for patients in the surgical repair group. During follow-up, two cases $(0.6 \%)$ of incarceration were observed in the watchful waiting group. O'Dwyer showed no differences between the two groups in pain at rest or at movement at 12 months followup. There also was no difference in QALY at 12 months. One case of acute hernia was observed (1.3\%) during follow-up.

\section{Discussion}

In this review of more than 10,000 patients we have critically appraised the appropriateness of surgery in patients with asymptomatic groin hernia repair. The rationale of repairing all groin hernias surgically, including asymptomatic hernias, is the possible risk of incarceration and strangulation. Surprisingly, how high this risk actually is remains unknown. An indirect parameter of this risk is the rate of emergency groin hernia repair, considering that emergency repairs are done in patients with either incarcerated or strangulated groin hernias. Multiple observational studies estimated this rate, finding an average of $7 \%$ of all groin hernia repairs as emergency repairs (see Table 2). Two recent randomized controlled studies by Fitzgibbons et al. and O'Dwyer et al. reported on the risk of incarceration in asymptomatic hernias [46, 49]. In both trials, patients with an asymptomatic inguinal hernia were randomized to either watchful waiting or elective repair. The incidence of incarceration in the watchful waiting group was 1.8 and 6.25 per 1,000 patients per year, respectively. These rates do not correspond with the average emergency rate of $7 \%$. An explanation for this difference can be that a considerable number of patients with a groin hernia do not consult a physician and do not undergo elective repair, resulting in a larger elective/emergency ratio. Recalling, the rationale of repairing all groin hernias surgically is the possible risk of incarceration and strangulation. This risk, due to the lack of evidence, has been overestimated and corresponds most likely to the rates reported by Fitzgibbons et al. and O'Dwyer et al. [46, 49].

The risk of incarceration and strangulation is increased by age over 60 years, femoral hernia site and short duration of signs. When a groin hernia does incarcerate or strangulate and emergency repair is required, the morbidity and mortality rates are higher compared to elective repair in socalled high-risk patients. Risk factors for increased morbidity and mortality are age above 49 years, delay between onset of symptoms and surgery greater than $12 \mathrm{~h}$, femoral hernia site, nonviable bowel and ASA-class 3 and 4. Therefore, an elective repair should be recommended to patients with an increased risk of incarceration and strangulation and to patients who are at risk for increased morbidity and mortality after emergency repair.

Different techniques are used during emergency repair. In low risk patients, tension-free mesh repair is a safe procedure [44, 45]. This technique is not associated with mesh-related complications, such as infections or increased recurrence rates compared to the use in elective setting. The long-term cure of a groin hernia repaired in an emergency setting is not inferior to elective repair in low-risk patients. In high risk patients, it is unclear which technique should be used during emergency groin hernia repair, and whether the use of mesh is contraindicated. More research should be done for future recommendations.

Elective groin hernia repair is considered a safe procedure. The short-term morbidity, the recurrence rate and the mortality rate are low. Nevertheless, over the past decade, reports have indicated high incidences of chronic pain after tension-free mesh hernia repair, ranging from 2 to $37 \%$ $[17,20-24]$. An inconsistency is observed, since these rates exceed the morbidity rates of $8 \%$ after elective groin hernia repair mentioned earlier (see Table 1). An explanation for this discrepancy and wide variety might be the lack of consensus about the definition of pain, the lack of assessment of chronic pain, or the lack of reporting chronic pain. Fitzgibbons et al. and O'Dwyer et al. both assessed pain in the surgical repair and in the watchful waiting group. Fitzgibbons showed that both groups reported less pain after 2 years compared to baseline [46]. O'Dwyer found that at 12 months there were no significant difference between 
both groups with regards to pain at rest or at movement [49]. Summarizing the two studies, results are indifferent towards surgical repair or watchful waiting in the treatment of an asymptomatic or minimal symptomatic groin hernia with regards to pain.

Fitzgibbons et al. [46] also assessed quality-of-life by means of the SF-36 and found a reduction in perception of pain unpleasantness for both groups. This reduction was significantly greater for patients in the surgical repair group. O'Dwyer et al. [49] found that general health status, one of the eight health concepts of the SF-36, increased for both the surgical and the observational arm over 12 months. The general health status in the surgical arm increased significantly more compared to the observational arm. Despite improvements in general health in the surgical arm, no significant difference in QALY was found at 12 months.

Stroupe et al. [47] calculated cost-effectiveness of surgical inguinal hernia repair and watchful waiting. They assessed that the incremental cost-effectiveness ratio for elective hernia repair is US $\$ 59,065 /$ QALY. A cost per QALY of approximately US $\$ 50,000$ is generally viewed as a reasonable cutoff for public funding of a medical procedure [48]. This means that elective hernia repair is hardly considered a worthwhile procedure, and that conservative treatment is a responsible approach from the viewpoint of cost-effectiveness. The calculations of Stroupe et al. were done in the United States, meaning that this analysis will be different for other health care systems and should be considered on a national level. O'Dwyer et al. [49] reported no difference in QALY for the surgical repair group compared to watchful waiting group but did report a difference in cost. Results show a difference in direct cost of $£ 402$ per patient for the operation group compared to the observation group at 574 days. Again, these costs were calculated in the United Kingdom and should be reviewed at a national level. In summary, surgical repair of an asymptomatic or minimal symptomatic inguinal hernia can hardly be considered a worthwhile procedure from the viewpoint of costeffectiveness.

It is estimated that approximately 70,000 hernia repairs are performed in Canada each year [50]. One-third of patients that present with a groin hernia is asymptomatic [38, 51]. This suggests that approximately 23,000 hernia repairs could be treated conservatively in Canada. Stroupe et al. [47] assessed the difference in costs between watchful waiting and elective repair to be US $\$ 1,831$. An amount of up to US $\$ 42,113,000$ could be saved annually. Considering the long waiting lists and the scarce resources in health care, watchful waiting should be suggested in every lowrisk patient with an asymptomatic groin hernia.

To date, two trials have reported on experience with watchful waiting in the treatment of asymptomatic or minimal symptomatic inguinal hernias [46, 49]. In both trials, patients in the watchful waiting group crossed over to the surgical repair group, mostly due to increase in pain. About $20 \%$ of asymptomatic or minimal symptomatic inguinal hernias will become symptomatic during watchful waiting, requiring surgical repair. The other $80 \%$ of patients with an asymptomatic or minimal symptomatic inguinal hernia will remain so. Approximately $1 \%$ of these patients will present with an incarcerated inguinal hernia requiring surgical repair.

Considering all outcomes, we propose an algorithm to help physicians assess whether a conservative approach is a safe approach towards a patient with an asymptomatic or minimal symptomatic groin hernia (Fig. 1). When a patient has had an asymptomatic or minimal symptomatic inguinal hernia for more than 3 months, is younger than 50 years and has an ASA class of 1 or 2, a conservative treatment is justified. When the inguinal hernia does incarcerate in such a patient, the hernia can be safely repaired with a tensionfree mesh technique. In patients with an asymptomatic or minimal symptomatic inguinal hernia for less than 3 months, who are older than 49 years or have an ASA class of 3 or 4 , an elective repair should be recommended. Patients with a femoral hernia should also be recommended elective repair. When the groin hernia does incarcerate in patients that fit the latter characteristics, it is unclear which technique should be used in emergency repair. Further research should be done to evaluate different techniques and outcomes in emergency hernia repair.

Some additional issues should be considered when implementing conservative treatment of an asymptomatic or minimal symptomatic inguinal hernia. Patients need to be well informed, understand the symptoms of incarceration and be instructed to come to hospital at the onset of symptoms. If the physician has the impression that the patient does not fulfill those requirements, the patient

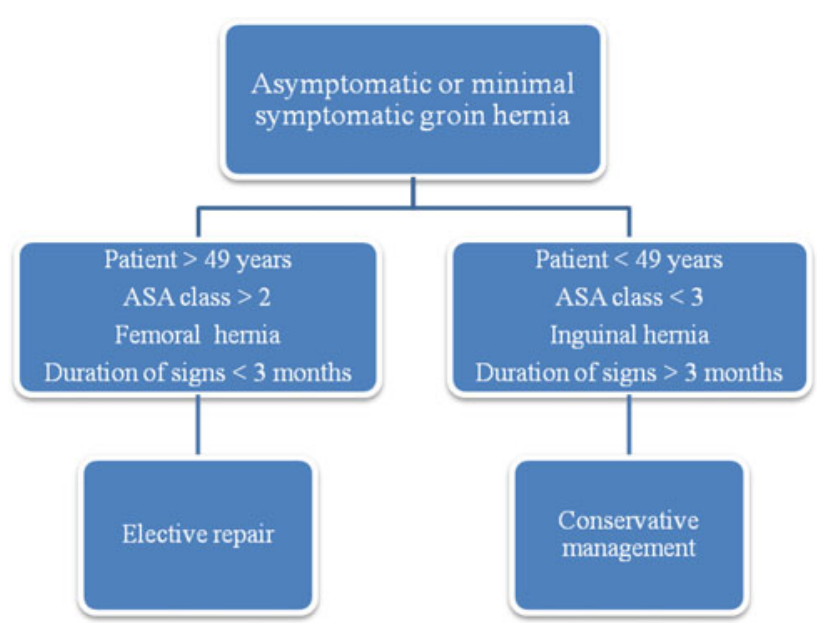

Fig. 1 Algorithm 
should be excluded from conservative treatment. Also, patients should live within a reasonable distance from a hospital, considering the significant increase in bowel resections in incarcerated hernias if duration is more than $12 \mathrm{~h}$. It is therefore important that the patient could reach a hospital within this timeframe.

Considering the best evidence available, a conservative treatment for asymptomatic or minimal symptomatic inguinal hernias is a safe and cost-effective treatment. Patients with an increased risk of incarceration or with an increased risk of higher morbidity and mortality after emergency repair should be excluded from conservative treatment. Nevertheless, prospective studies are needed to improve recommendations.

\section{References}

1. Kingsnorth A, LeBlanc K (2003) Hernias: inguinal and incisional. Lancet 362(9395):1561-1571

2. Rutkow IM (2003) Demographic and socioeconomic aspects of hernia repair in the United States in 2003. Surg Clin North Am 83(5):1045-1051, v-vi

3. Malek S, Torella F, Edwards PR (2004) Emergency repair of groin herniae: outcome and implications for elective surgery waiting times. Int J Clin Pract 58(2):207-209

4. Palumbo LT, Sharpe WS (1971) Primary inguinal hernioplasty in the adult. Surg Clin N Am 51(6):1293-1307

5. McIntosh A, Hutchinson A, Roberts A, Withers H (2000) Evidence-based management of groin hernia in primary care-a systematic review. Fam Pract 17(5):442-447

6. Neutra R, Velez A, Ferrada R, Galan R (1981) Risk of incarceration of inguinal hernia in Cali Colombia. J Chronic Dis 34(11): 561-564

7. Primatesta P, Goldacre MJ (1996) Inguinal hernia repair: incidence of elective and emergency surgery, readmission and mortality. Int J Epidemiol 25(4):835-839

8. Nilsson E, Kald A, Anderberg B et al (1997) Hernia surgery in a defined population: a prospective three year audit. Eur J Surg 163(11):823-829

9. Andrews NJ (1981) Presentation and outcome of strangulated external hernia in a district general hospital. Br J Surg 68(5): 329-332

10. Rai S, Chandra SS, Smile SR (1998) A study of the risk of strangulation and obstruction in groin hernias. Aust N Z J Surg 68(9):650-654

11. Gallegos NC, Dawson J, Jarvis M, Hobsley M (1991) Risk of strangulation in groin hernias. Br J Surg 78(10):1171-1173

12. Ponka JL, Brush BE (1974) Experiences with the repair of groin hernia in 200 patients aged 70 or older. J Am Geriatr Soc 22(1):18-24

13. Oishi SN, Page CP, Schwesinger WH (1991) Complicated presentations of groin hernias. Am J Surg 162(6):568-570

14. Kulah B, Kulacoglu IH, Oruc MT et al (2001) Presentation and outcome of incarcerated external hernias in adults. Am J Surg 181(2):101-104

15. Hair A, Paterson C, Wright D, Baxter JN, O'Dwyer PJ (2001) What effect does the duration of an inguinal hernia have on patient symptoms? J Am Coll Surg 193(2):125-129

16. Schumpelick V, Treutner KH, Arlt G (1994) Inguinal hernia repair in adults. Lancet 344(8919):375-379
17. Eklund A, Montgomery A, Bergkvist L, Rudberg C (2010) Chronic pain 5 years after randomized comparison of laparoscopic and Lichtenstein inguinal hernia repair. Br J Surg 97(4):600-608

18. Kehlet H, Bay-Nielsen M, Kingsnorth A (2002) Chronic postherniorrhaphy pain-a call for uniform assessment. Hernia 6(4):178-181

19. Cunningham J, Temple WJ, Mitchell P, Nixon JA, Preshaw RM, Hagen NA (1996) Cooperative hernia study. Pain in the postrepair patient. Ann Surg 224(5):598-602

20. McCormack K, Scott N, Go PMNYH, Ross SJ, Grant A, Collaboration the EU Hernia Trialists (2003) Laparoscopic techniques versus open techniques for inguinal hernia repair. Cochrane Database Syst Rev 1. Art. No.:CD001785. doi:10.1002/14651858.CD001785

21. Bay-Nielsen M, Perkins FM, Kehlet H (2003) Pain and functional impairment 1 year after inguinal herniorrhaphy: a nationwide questionnaire study. Ann Surg 233(1):1-7

22. The MRC Laparoscopic Groin Hernia Trial Group (1999) Laparoscopic versus open repair of groin hernia: a randomised comparison. Lancet 354(9174):185-190

23. Wright D, Paterson C, Scott N, Hair A, O'Dwyer PJ (2002) Five-year follow-up of patients undergoing laparoscopic or open groin hernia repair: a randomized controlled trial. Ann Surg 235(3):333-337

24. Langeveld HR, van't Riet M, Weidema WF, Stassen LP, Steyerberg EW, Lange J, Bonjer HJ, Jeekel J (2010) Total extraperitoneal inguinal hernia repair compared with Lichtenstein (the LEVEL-Trial): a randomized controlled trial. Ann Surg 251(5):819-824

25. Lichtenstein IL (1987) Herniorrhaphy. A personal experience with 6,321 cases. Am J Surg 153(6):553-559

26. Palumbo LT, Mighell SJ (1954) Primary inguinal hernioplasty in geriatrics. Geriatrics 9(1):8-14

27. Bendavid R (1998) Complications of groin hernia surgery. Surg Clin N Am 78(6):1089-1103

28. Vrijland WW, van den Tol MP, Luijendijk RW et al (2002) Randomized clinical trial of non-mesh versus mesh repair of primary inguinal hernia. Br J Surg 89(3):293-297

29. Scott NW, McCormack K, Graham P, Go PM, Ross SJ, Grant AM (2002) Open mesh versus non-mesh for repair of femoral and inguinal hernia. Cochrane Database Syst Rev 4. Art. No.:CD002197. doi:10.1002/14651858.CD002197

30. Stylianidis G, Haapamaki MM, Sund M, Nilsson E, Nordin P (2010) Management of the hernial sac in inguinal hernia repair. $\mathrm{Br}$ J Surg 97(3):415-419

31. Eklund AS, Montgomery AK, Rasmussen IC, Sandbue RP, Bergkvist LA, Rudberg CR (2009) Low recurrence rate after laparoscopic (TEP) and open (Lichtenstein) inguinal hernia repair: a randomized, multicenter trial with 5-year follow-up. Ann Surg 249(1):33-38

32. Nilsson H, Stylianidis G, Haapamaki M, Nilsson E, Nordin P (2007) Mortality after groin hernia surgery. Ann Surg 245(4):656-660

33. Alvarez JA, Baldonedo RF, Bear IG, Solis JA, Alvarez P, Jorge JI (2004) Incarcerated groin hernias in adults: presentation and outcome. Hernia 8(2):121-126

34. Lewis DC, Moran CG, Vellacott KD (1989) Inguinal hernia repair in the elderly. J R Coll Surg Edinburgh 34(2):101-103

35. Brasso K, Londal Nielsen K, Christiansen J (1989) Long-term results of surgery for incarcerated groin hernia. Acta Chir Scand 155(11-12):583-585

36. McEntee G, Pender D, Mulvin D et al (1987) Current spectrum of intestinal obstruction. Br J Surg 74(11):976-980

37. Kulah B, Duzgun AP, Moran M, Kulacoglu IH, Ozmen MM, Coskun F (2001) Emergency hernia repairs in elderly patients. Am J Surg 182(5):455-459

38. Ohana G, Manevwitch I, Weil R et al (2004) Inguinal hernia: challenging the traditional indication for surgery in asymptomatic patients. Hernia 8(2):117-120 
39. Tingwald GR, Cooperman M (1982) Inguinal and femoral hernia repair in geriatric patients. Surg Gynecol Obstet 154(5):704-706

40. Williams JS, Hale HW (1966) The advisability of inguinal herniorrhaphy in the elderly. Surg Gynecol Obstet 122(1):100-104

41. Kurt N, Oncel M, Ozkan Z, Bingul S (2003) Risk and outcome of bowel resection in patients with incarcerated groin hernias: retrospective study. World J Surg 27(6):741-743

42. Kauffman HM Jr, O’Brien DP (1970) Selective reduction of incarcerated inguinal hernia. Am J Surg 119(6):660-673

43. Bekoe S (1973) Prospective analysis of the management of incarcerated and strangulated inguinal hernias. Am J Surg 126(5):665-668

44. Elsebae MM, Nasr M, Said M (2008) Tension-free repair versus Bassini technique for strangulated inguinal hernia: a controlled randomized study. Int J Surg 6(4):302-305

45. Bessa SS, Katri KM, Abdel-Salam WN, Abdel-Baki NA (2007) Early results from the use of the Lichtenstein repair in the management of strangulated groin hernia. Hernia 11(3):239-242

46. Fitzgibbons RJ Jr, Giobbie-Hurder A, Gibbs JO et al (2006) Watchful waiting vs repair of inguinal hernia in minimally symptomatic men: a randomized clinical trial. JAMA 295(3):285-292

47. Stroupe KT, Manheim LM, Luo P et al (2006) Tension-free repair versus watchful waiting for men with asymptomatic or minimally symptomatic inguinal hernias: a cost-effectiveness analysis. J Am Coll Surg 203(4):458-468
48. Briggs A, Gray A (2000) Using cost effectiveness information. BMJ 320(7229):246

49. O'Dwyer PJ, Norrie J, Alani A, Walker A, Duffy F, Horgan P (2006) Observation or operation for patients with an asymptomatic inguinal hernia: a randomized clinical trial. Ann Surg 244(2): 167-173

50. Poulin EC, Marcaccio MJ (1997) Symposium on the management of inguinal hernias. 1. Introduction. Can J Surg 40(3):185

51. O'Riordan DC, Kingsnorth AN (1998) Audit of patient outcomes after herniorrhaphy. Surg Clin North Am 78(6):1129-1139, viii-ix

52. Nehme AE (1983) Groin hernias in elderly patients. Management and prognosis. Am J Surg 146(2):257-260

53. Allen PI, Zager M, Goldman M (1987) Elective repair of groin hernias in the elderly. Br J Surg 74(11):987

54. Haapeniemi S, Sandblom G, Nilsson E (1999) Mortality after elective and emergency surgery for inguinal and femoral hernia. Hernia 4:205-208

55. Nilsson E, Haapaniemi S (1998) Hernia registers and specialization. Surg Clin N Am 78(6):1141-1155

56. Hair A, Duffy K, McLean J et al (2000) Groin hernia repair in Scotland. Br J Surg 87(12):1722-1726

57. Askew G, Williams GT, Brown SC (1992) Delay in presentation and misdiagnosis of strangulated hernia: prospective study. J R Coll Surg Edinburgh 37(1):37-38 